\title{
Inflammatory Bowel Disease, Are Symptoms and Diet Linked? ${ }^{+}$
}

\author{
Hannah Morton ${ }^{1, *}$, Kevin C. Pedley ${ }^{2}$, Robin J. C. Stewart ${ }^{3}$ and Jane Coad ${ }^{1}$ \\ 1 School of Food \& Advanced Technology, Massey University, Palmerston North 4474, New Zealand; \\ j.coad@massey.ac.nz \\ 2 School of Health Sciences, Massey University, Palmerston North 4474, New Zealand; \\ k.c.pedley@massey.ac.nz \\ 3 School of Applied Science, Universal College of Learning, Palmerston North 4474, New Zealand; \\ b.stewart@ucol.ac.nz \\ * Correspondence: h.morton1@massey.ac.nz; Tel.: +64-27-314-0599 \\ + Presented at the 2019 Annual Meeting of the Nutrition Society of New Zealand, Napier, New Zealand, \\ 28-29 November 2019.
}

Published: 13 December 2019

New Zealand (NZ) has one of the highest rates of Inflammatory Bowel Disease (IBD), a collective term for three chronic inflammatory conditions that affect the gastrointestinal tract. The two mostly frequently diagnosed of these conditions are Crohn's Disease (CD) and Ulcerative Colitis (UC). Many IBD patients believe certain foods influence their disease symptoms and consequently reduce or avoid consumption of these foods. The objective of this study was to identify foods, additives and cooking methods associated with the reduction or exacerbation of symptoms in NZ IBD patients.

231 participants aged 16 years and above, with clinically diagnosed IBD, were recruited throughout NZ. Participants completed a self-administered questionnaire concerning the reduction or exacerbation of their symptoms and 100 often-implicated foods, 7 additives, and 4 cooking methods. Participants rated the effect of each dietary element as 'little', 'moderate', or 'definite'.

Foods, additives, and/or cooking methods were associated with symptom reduction in $84(36 \%)$ of participants, and with symptom exacerbation in 149 (64\%) of participants. The most frequently reported foods to reduce symptoms (definite effect) were rice (10.7\%), white bread (10.7), and banana (9.5); while those reported to exacerbate symptoms (definite effect) were deep frying foods $(34 \%)$, chilli (32\%) and full-grain bread (31\%). The number of patients reporting a dietary association with the reduction or exacerbation of symptoms was similar, $36 \%$ and $6 \%$ of CD patients, and $33 \%$ and $67 \%$ of UC patients. However, the number of dietary elements reported to have an effect was greater per UC patient.

The reported association between dietary elements and IBD symptoms may be attributable to ease of digestion, an effect on intestinal permeability, or in the case of symptom exacerbation, a genuine food intolerance. Further research is warranted to investigate the mechanism/s involved, and may lead to the development of an IBD friendly diet.

(C) 2019 by the authors. Licensee MDPI, Basel, Switzerland. This article is an open access article distributed under the terms and conditions of the Creative Commons Attribution (CC BY) license (http://creativecommons.org/licenses/by/4.0/). 